\title{
Bäcklund Transformations of Some Nonlinear Evolution Equations VIA Painlevè Analysis
}

\author{
M. F. El-Sabbagh, R. Zait, and R. M. Abdelazeem \\ Mathematics Department, Faculty of Science, Minia university, Egypt
}

\begin{abstract}
In this paper we present the Painlevè test for the $(1+1)$-dimensional travelling regularized long wave (TRLW) equation, the (2+1)-dimensional Boiti-Leon-Manna-Pempinelli (BLMP) equation, the modified improved Kadomtsev-Petviashvili equation (MIKP) and the variant shallow water wave equations. The associated Bäcklund transformations are obtained directly from the Painlevè test.

Keywords: the $(1+1)$-dimensional travelling regularized long wave (TRLW) equation, the (2+1)-dimensional Boiti-Leon-Manna-Pempinelli (BLMP) equation, the modified improved Kadomtsev-Petviashvili equation $(M I K P)$, the variant shallow water wave equations and Painlevè analysis.
\end{abstract}

\section{Introduction}

Painlevè analysis is a powerful tool in investigating the integrability properties of differential equations. For systems with the painlevè property, Bäcklund transformations can be defined. These appear as truncations of certain expansions of solutions about its singular manifold. Many methods have been established to study characteristic properties for integrable NLEEs and their interrelations. Some of the most important methods are the inverse scattering method (IST) [1-4], the Bäcklund transformations [5, 6] and the Painlevè analysis method [7-12]. If a PDE which has no points such as movable branch, algebraic and logarithmic then is called P-type. An ordinary differential equation (ODE) might still admit movable essential singularities without movable branch points. This method does not identify essential singularities and therefore it provides only necessary conditions for an ODE to be of P-type. Singularity structure analysis admitting the P-property advocated by Ablowitz et al. for ODEs and extended to PDE by Weiss, Tabor and Carnevale (WTC), plays a key role of investigating the integrability properties of many NLEEs. The well-known procedure of WTC requires,

- The determination of leading orders Laurent series,

- The identification of powers at which the arbitrary functions can enter into the Laurent series called resonances,

- Verifying that, at the resonance values, sufficient number of arbitrary functions exist without introducing the movable critical manifold.

According to the WTC method, the general solution of PDE is in the form

$$
u(x, t)=\phi^{\alpha}(x, t) \sum_{j=0}^{\infty} u_{j}(x, t) \phi^{j}(x, t)
$$

where $\alpha$ is a negative integer determined by balancing the powers of $\varphi$ of dominant terms in the equation. $\varphi$ is a non-characteristic manifold, $\varphi(x, t)=0$ is the equation of singular manifold. The functions $u_{j}(j=o, i, 2, \ldots)$ have to be determined by substitution of expansion (1.1) into the PDE, so it becomes:

$$
\sum_{j=0}^{\infty} E_{j}\left(u_{0}, . . u_{j}, \varphi\right) \varphi^{j+q}(x, t)=0 \text {, }
$$

where $q$ is some negative constant, $E_{j}$ depends on $\phi$ only by the derivatives of $\phi$. The successive practical steps of Painleve' analysis are the following:

- Determine the possible leading orders $p$ by balancing two or more terms of the PDE and expressing that they dominate the other terms.

- Solve equation $E_{0}=0$ for non-zero values of $u_{0}$; this may lead to several solutions, called branches.

- Find the resonances, i.e. the values of $k$ for which $u_{k}$ cannot be determined from equation $E_{j}=0$.

This last equation has generally the form

$$
E_{j}=(j+1) P(j) \phi_{x}^{j} \phi_{t}^{n-2} u_{j}+Q\left(u_{0}, \ldots u_{j-1}, \phi\right)=0, \quad \mathrm{j}>0
$$


where $\mathrm{n}$ is the order of the PDE, $0 \leq \mathrm{j} \leq \mathrm{n}$ and $p$ is a polynomial of degree $n-1$. The values of the resonances are the zeros of $p$.

- Determine whether the resonances are 'compatible' or not. At resonance, after substitution in (1.3) of the previously computed $u_{i}, i \leq j-1$, the function $Q$ is either zero or non-zero then in the case $u_{j}$ can arbitrarily be chosen and the expansion (1.1) does not exist for arbitrary $\phi$, so the resonance is called compatible.

\section{The (1+1) -dimensional travelling regularized long wave (TRLW) equation}

Consider the $(1+1)$-dimensional travelling regularized long wave equation [13]:

$u_{t}+u_{x}+\beta u u_{x}+u_{x t t}=0$

We first present the Painlevè test of the TRLW equation. The leading order of solution of Eq. (2.1) is assumed as

$u \approx u_{0} \varphi^{\alpha}$

Substituting Eq. (2.2) into (2.1) and equating the most dominant terms, the following results are obtained

$u_{0}=\frac{-12}{\beta} \phi_{t}^{2}, \quad \alpha=-2$

For finding the resonances, the full Laurent series

$u=u_{0} \varphi^{-2}+\sum_{j=1}^{\infty} u_{j} \varphi^{j-2}$

is substituted into Eq. (2.1) and by equating the coefficients of $\phi^{j-5}$, the polynomial equation in $j$ is found as

$j^{3}-9 j^{2}+14 j+24=0$

Using Eq. (2.5), the resonances are found to be

$j=-1,4,6$

As usual, the resonance at $j=-1$ corresponds to the arbitrariness of singular manifold $\varphi(x, t)=0$. In order to check the existence of sufficient number of arbitrary functions at the other resonance values, the full Laurent expansion (2.4) is substituted in Eq. (2.1). From the coefficient of $\phi^{-5}$, the explicit value of $u_{0}$ is obtained as given in Eq. (2.3).

To construct the Bäcklund transformation of Eq. (2.1), let us truncate the Laurent series at the constant level term to give

$u=u_{0} \phi^{-2}+u_{1} \phi^{-1}$

Hence

$u=\frac{-12 \phi_{t}^{2}}{\beta \phi^{2}}+\frac{u_{1}}{\phi}$,

where the pair of function $\left(u, u_{1}\right)$ satisfy Eq. (2.1), and hence Eq. (2.8) is the associated Bäcklund

transformation of Eq. (2.1) relating a solution $u$ with a known solution $u_{1}$ of the Eq. (2.1) which can be taken to be so.

We can also construct another Bäcklund transformation of Eq. (2.1) to be

$u=\frac{u_{0}}{\phi^{2}}+\frac{u_{1}}{\phi}+u_{2}$,

where $u, u_{1}$ satisfy Eq. (2.1) while $u_{2}$ is given by:

$u_{2}=\frac{1}{-24 \phi_{t}^{2} \phi_{x}}\left(-2 u_{0 x} \phi_{t t}+\beta u_{1} u_{0 x}-\beta u_{1}^{2} \phi_{x}-12 \phi_{t}^{2} u_{1 x}-2 u_{0} \phi_{t t x}+2 u_{1} \phi_{x} \phi_{t t}-\right.$

$\left.2 u_{0} \phi_{x}-4 u_{0 t} \phi_{x t}-4 u_{0 x t} \phi_{t}-2 u_{0 t t} \phi_{x}+4 u_{1 t} \phi_{x} \phi_{t}-2 u_{0} \phi_{t}+4 u_{1} \phi_{x t} \phi_{t}+2 u_{1 x} \phi_{t}^{2}\right)$,

and hence Eq. (2.10) is a Bäcklund transformation too. Also let's truncate the Laurent series again we get 
$u=\frac{6 \phi_{x}^{2}}{\phi^{2}}+\frac{u_{1}}{\phi}+u_{2}+u_{3} \varphi$,

where $u, u_{1}$ satisfy Eq. (2.1) and $u_{2}$ is given by Eq. (2.10) $u_{3}$ is given by:

$u_{3}=\frac{1}{-12 \phi_{x} \phi_{t}^{2}}\left(-u_{1} \phi_{x}+\beta u_{2} u_{0 x}-\beta u_{2} u_{1} \phi_{x}-u_{1} \phi_{x t t}+\beta u_{2 x} u_{0}-u_{1} \phi_{t}+\beta u_{1 x} u_{1}-\right.$

$\left.u_{1 t t} \phi_{x}+u_{0 t}-2 u_{1 x t} \phi_{t}+u_{0 t t x}+u_{0 x}-2 u_{1 t} \phi_{x t}-u_{1 x} \phi_{t t}\right)$

We can make more truncations to the Laurent series at the constant level term $u_{4}$ and it will produce another Bäcklund transformation for Eq. (2.1).

\section{The (2+1)-dimensional Boiti-Leon-Manna-Pempinelli (BLMP) equation}

Consider the (2+1)-dimensional Boiti-Leon-Manna-Pempinelli (BLMP) equation [14-17]:

$u_{y t}-3 u_{x y} u_{x}-3 u_{x x} u_{y}+u_{x x y}=0$

We first present the Painlevè test of the BLMP equation. The leading order of solution of Eq. (3.1) is assumed as $u \approx u_{0} \varphi^{\alpha}$

Substituting Eq. (3.2) into (3.1) and equating the most dominant terms, the following results are obtained

$u_{0}=-2 \phi_{x}, \quad \alpha=-1$

For finding the resonances, the full Laurent series.

$u=u_{0} \varphi^{-1}+\sum_{j=1}^{\infty} u_{j} \varphi^{j-1}$

is substituted into Eq. (3.1) and by equating the coefficients of $\phi^{j-5}$, the polynomial equation in $j$ is found as

$j^{4}-10 j^{3}+23 j^{2}+10 j-24=0$

Using Eq. (3.5), the resonances are found to be

$j=-1,1,4,6$

As usual, the resonance at $j=-1$ corresponds to the arbitrariness of singular manifold $\varphi(x, y, t)=0$. In order to check the existence of sufficient number of arbitrary functions at the other resonance values, the full Laurent expansion (3.4) is substituted in Eq. (3.1). From the coefficient of $\phi^{-5}$, the explicit value of $u_{0}$ is obtained as given in Eq. (3.3).

To construct the Bäcklund transformation of Eq. (3.1), let us truncate the Laurent series at the constant level term to give

$u=u_{0} \varphi^{-1}+u_{1}$

Hence

$u=\frac{-2 \varphi_{x}}{\varphi}+u_{1}$

where the pair of function $\left(u, u_{1}\right)$ satisfy Eq. (3.1) and hence Eq. (3.8) is the associated Bäcklund transformation of Eq. (3.1) relating a solution $u$ with a known solution $u_{1}$ of the Eq. (3.1) which can be taken to be so.

We can also construct another Bäcklund transformation of Eq. (3.1) to be

$u=\frac{-2 \phi_{x}}{\phi}+u_{1}+u_{2} \varphi$,

where $u, u_{1}$ satisfy Eq. (3.1) while $u_{2}$ is given by: 


$$
\begin{aligned}
& u_{2}=\frac{1}{-24 \phi_{x}^{3} \phi_{y}}\left(-12 \phi_{x}^{2} \phi_{x x y}+6 u_{0 x x} \phi_{x} \phi_{y}+12 u_{0 x} \phi_{x} \phi_{x y}+6 u_{0 x y} \phi_{x}^{2}+12 \phi_{x}^{3} u_{1 y}-4 \phi_{x} \phi_{y} \phi_{t}-\right. \\
& 6 u_{0 x x} \phi_{x} \phi_{y}+9 u_{0 y} u_{0 x} \phi_{x}-12 \phi_{x} \phi_{x y} \phi_{x x}+6 u_{0 x} \phi_{x x} \phi_{y}+12 \phi_{x}^{2} \phi_{y} u_{1 x}-6 \phi_{x}^{2} u_{0 x y} \\
& \left.-6 \phi_{x} \phi_{x y} u_{0 x}+3 u_{0 x}^{2} \phi_{y}-4 \phi_{x x x} \phi_{x} \phi_{y}\right),
\end{aligned}
$$

and hence Eq. (3.9) is a Bäcklund transformation too. Also let's truncate the Laurent series again we get

$u=\frac{-2 \phi_{x}}{\phi}+u_{1}+u_{2} \varphi+u_{3} \varphi^{2}$,

where $u, u_{1}$ satisfy Eq. (3.1) and $u_{2}$ is given by Eq. (3.10), while $u_{3}$ is given by:

$$
\begin{aligned}
& \mathrm{u}_{3}=\frac{-1}{24 \phi_{x}^{3} \phi_{y}}\left(3 \mathrm{u}_{0} \phi_{x x} \mathrm{u}_{1 y}-\mathrm{u}_{0 y} \phi_{t}+6 \mathrm{u}_{0 x} \phi_{x} \mathrm{u}_{1 y}+3 \mathrm{u}_{0} \phi_{x y} \mathrm{u}_{1 x}-3 \mathrm{u}_{0} \phi_{x}^{2} \mathrm{u}_{2 y}-\mathrm{u}_{0 t} \phi_{y}-\mathrm{u}_{0} \phi_{y t}\right. \\
& +3 \mathrm{u}_{0 y} \phi_{x} \mathrm{u}_{1 x}+3 \mathrm{u}_{0 x} \phi_{y} \mathrm{u}_{1 x}+3 \mathrm{u}_{0} \phi_{x} \mathrm{u}_{1 x y}+3 \mathrm{u}_{0 y} \phi_{x}^{2} \mathrm{u}_{2}+3 \mathrm{u}_{0} \phi_{y} \mathrm{u}_{1 x x}-\mathrm{u}_{0 y} \phi_{x x x}-3 \mathrm{u}_{0 x x} \phi_{x y}- \\
& \mathrm{u}_{0 x x x} \phi_{y}-3 \mathrm{u}_{0 x} \phi_{x x y}-3 \mathrm{u}_{0 x x y} \phi_{x}-3 \mathrm{u}_{0 x y} \phi_{x x}-\mathrm{u}_{0} \phi_{x x x y}-3 \mathrm{u}_{0 x y} \mathrm{u}_{0 x}-3 \mathrm{u}_{0 x x} \mathrm{u}_{0 y}+3 \mathrm{u}_{0} \phi_{x} \phi_{y} \mathrm{u}_{2 x} \\
& \left.+6 \mathrm{u}_{0} \phi_{x x} \mathrm{u}_{2} \phi_{y}+9 \mathrm{u}_{0 x} \phi_{x} \mathrm{u}_{2} \phi_{y}+6 \mathrm{u}_{0} \phi_{x y} \mathrm{u}_{2} \phi_{x}\right)
\end{aligned}
$$

We can make more truncations to the Laurent series at the constant level term $u_{4}$ and it will produce another Bäcklund transformation for Eq. (3.1).

\section{The modified improved Kadomtsev-Petviashvili equation (MIKP)}

Consider the modified improved Kadomtsev-Petviashvili equation (MIKP) [18, 19]:

$u_{t x}+2 u u_{x}^{2}+u^{2} u_{x x}+a u_{x x x x}+b u_{y y}=0$

We first present the Painlevè test of the MIKP equation. The leading order of solution of Eq. (4.1) is assumed as:

$u \approx u_{0} \varphi^{\alpha}$

Substituting Eq. (4.2) into (4.1) and equating the most dominant terms, the following results are obtained

$u_{0}=\sqrt{-6 a} \phi_{x}, \quad \alpha=-1$

For finding the resonances, the full Laurent series.

$u=u_{0} \varphi^{-1}+\sum_{j=1}^{\infty} u_{j} \varphi^{j-1}$

is substituted into Eq. (4.1) and by equating the coefficients of $\phi^{j-5}$, the polynomial equation in $j$ is found as:

$j^{4}-10 j^{3}+29 j^{2}-8 j-48=0$

Using Eq. (4.5), the resonances are found to be $j=-1,3,4,4$

As usual, the resonance at $j=-1$ corresponds to the arbitrariness of singular manifold $\varphi(x, y, t)=0$. In order to check the existence of sufficient number of arbitrary functions at the other resonance values, the full Laurent expansion (4.4) is substituted in Eq. (4.1). From the coefficient of $\phi^{-5}$, the explicit value of $u_{0}$ is obtained as given in Eq. (4.3).

To construct the Bäcklund transformation of Eq. (4.1), let us truncate the Laurent series at the constant level term to give

$u=u_{0} \varphi^{-1}+u_{1}$

Hence

$u=\frac{\sqrt{-6 a} \varphi_{x}}{\varphi}+u_{1}$, 
where the pair of function $\left(u, u_{1}\right)$ satisfy Eq. (4.1) and hence Eq. (4.8) is the associated Bäcklund transformation of Eq. (4.1) relating a solution $u$ with a known solution $u_{1}$ of the Eq. (4.1) which can be taken to be so.

We can also construct another Bäcklund transformation of Eq. (4.1) to be

$u=\frac{\sqrt{-6 a} \phi_{x}}{\phi}+u_{1}+u_{2} \varphi$

where $u, u_{1}$ satisfy Eq. (4.1) while $u_{2}$ is given by:

$$
\begin{aligned}
& u_{2}=\frac{1}{12 a \phi_{x}^{4}}\left(-6 a \phi_{x}^{2} u_{0 x x}+12 a \phi_{x}^{2} u_{1} \phi_{x x}-8 \sqrt{-6 a} \phi_{x}^{2} u_{1} u_{0 x}+2 \sqrt{-6 a} u_{1}^{2} \phi_{x}^{3}+2 \sqrt{-6 a} \phi_{x}^{2} \phi_{t}\right. \\
& +8 a \sqrt{-6 a} \phi_{x}^{2} \phi_{x x x}+12 a u_{0 x x} \phi_{x}^{2}+6 a \sqrt{-6 a} \phi_{x}^{3}+24 a u_{0 x} \phi_{x} \phi_{x x}+2 \sqrt{-6 a} b \phi_{x} \phi_{y}^{2}+24 a \phi_{x}^{3} u_{1 x} \\
& \left.+2 \sqrt{-6 a} \phi_{x} u_{o x}^{2}\right),
\end{aligned}
$$

and hence Eq. (4.10) is a Bäcklund transformation too.

\section{The variant shallow water wave equations}

Consider the variant shallow water wave equations [20]:

$$
\begin{aligned}
& u_{t}+v_{x}+u u_{x}-\varepsilon^{2} u_{x x t}=0, \\
& v_{t}+u_{x}+(u v)_{x}+\delta^{2} u_{x x x}=0
\end{aligned}
$$

We first present the Painlevè test of the variant shallow water wave equations. The leading order of solution of system (5.1) is assumed as:

$u \approx u_{0} \varphi^{\alpha}, \quad v \approx v_{0} \varphi^{\beta}$

Substituting Eq. (5.2) into (5.1) and equating the most dominant terms, the following results are obtained

$u_{0}=12 \varepsilon^{2} \phi_{x} \phi_{t}, \quad v_{0}=-6 \delta^{2} \phi_{x}^{2}, \quad \alpha=\beta=-2$

For finding the resonances, the full Laurent series.

$u=u_{0} \varphi^{-2}+\sum_{j=1}^{\infty} u_{j} \varphi^{j-2}$

$v=v_{0} \varphi^{-2}+\sum_{j=1}^{\infty} v_{j} \varphi^{j-2}$

is substituted into system (5.1) and by equating the coefficients of $\phi^{j-5}$, the polynomial equation in $j$ is found as:

$j^{3}-9 j^{2}+14 j+24=0$

Using Eq. (5.5), the resonances are found to be

$j=-1,4,6$

As usual, the resonance at $j=-1$ corresponds to the arbitrariness of singular manifold $\varphi(x, t)=0$. In order to check the existence of sufficient number of arbitrary functions at the other resonance values, the full Laurent expansion (5.4) is substituted in system (5.1). From the coefficient of $\phi^{-5}$, the explicit value of $u_{0}, v_{0}$ is obtained as given in Eq. (5.3).

To construct the Bäcklund transformation of system (5.1), let us truncate the Laurent series at the constant level term to give

$u=u_{0} \varphi^{-2}+u_{1} \phi^{-1}+u_{2}, \quad v=v_{0} \varphi^{-2}+v_{1} \phi^{-1}+v_{2}$,

Hence

$u=\frac{12 \varepsilon^{2} \phi_{x} \phi_{t}}{\phi^{2}}+\frac{u_{1}}{\phi}+u_{2}, \quad v=\frac{-6 \delta^{2} \phi_{x}^{2}}{\phi^{2}}+\frac{v_{1}}{\phi}+v_{2}$, 
where the pair of function $(u, v)$ and $\left(u_{1}, v_{1}\right)$ satisfy system (5.1), while $u_{2}, v_{2}$ satisfy respectively the following equations:

$$
\begin{aligned}
& u_{2}=\frac{1}{24 \varepsilon^{2} \phi_{x}^{2} \phi_{t}}\left(2 \varepsilon^{2} u_{0 x x} \phi_{t}+24 \varepsilon^{4} \phi_{x} \phi_{t} \phi_{t x x}-4 \varepsilon^{2} u_{1 x} \phi_{x} \phi_{t}-4 \varepsilon^{2} u_{1} \phi_{x} \phi_{t x}+4 \varepsilon^{2} u_{0 x} \phi_{t x}+\right. \\
& 2 \varepsilon^{2} u_{0 t} \phi_{x x}-2 \varepsilon^{2} u_{1} \phi_{x x} \phi_{t}-2 \varepsilon^{2} u_{1 t} \phi_{x}^{2}+4 \varepsilon^{2} u_{0 t x} \phi_{x}-24 \varepsilon^{2} \phi_{x} \phi_{t}^{2}+12 \varepsilon^{2} \phi_{x} \phi_{t} u_{1 x}+u_{1} u_{0 x} \\
& \left.-u_{1}^{2} \phi_{x}+12 \delta^{2} \phi_{x}^{3}\right), \\
& v_{2}=\frac{-1}{24 \varepsilon^{2} \phi_{x}^{2} \phi_{t}}\left(24 \varepsilon^{2} \phi_{x}^{2} \phi_{t}+24 \delta^{2} \varepsilon^{2} \phi_{x} \phi_{t} \phi_{x x x}+6 \delta^{2} u_{0 x} \phi_{x x}+6 \delta^{2} u_{0 x x} \phi_{x}-6 \delta^{2} u_{1} \phi_{x} \phi_{x x}\right. \\
& \left.-6 \delta^{2} u_{1 x} \phi_{x}^{2}-12 \delta^{2} \phi_{x}^{3} u_{2}+2 \phi_{x} v_{1} u_{1}-v_{1} u_{o x}-v_{0 x} u_{1}+6 \delta^{2} \phi_{x}^{2} u_{1 x}-12 \varepsilon^{2} \phi_{x} \phi_{t} v_{1 x}-12 \delta^{2} \phi_{x}^{2} \phi_{t}\right),
\end{aligned}
$$

and hence Eq. (5.8) is the associated Bäcklund transformation of system (5.1).

We can also construct another Bäcklund transformation of system (5.1) to be

$$
u=\frac{u_{0}}{\phi^{2}}+\frac{u_{1}}{\phi}+u_{2}+u_{3} \phi+u_{4} \phi^{2}, \quad v=\frac{v_{0}}{\phi^{2}}+\frac{v_{1}}{\phi}+v_{2}+v_{3} \phi+v_{4} \phi^{2},
$$

where the pair of function $(u, v)$ and $\left(u_{4}, v_{4}\right)$ satisfy system (5.1) while $u_{2}$ and $v_{2}$ is given by Eq. (5.9) and Eq. (5.10) respectively but $u_{3}$ and $v_{3}$ are given respectively by the following equations:

$$
\begin{aligned}
& u_{3}=\frac{1}{12 \varepsilon^{2} \phi_{x}^{2} \phi_{t}}\left(u_{0 t}-u_{1} \phi_{t}+\varepsilon^{2} u_{1 t} \phi_{x x}+\varepsilon^{2} u_{1} \phi_{t x x}+2 \varepsilon^{2} u_{1 x} \phi_{t x}-\varepsilon^{2} u_{0 t x x}+\varepsilon^{2} u_{1 x x} \phi_{t}\right. \\
& \left.+2 \varepsilon^{2} u_{1 t x} \phi_{x}+v_{0 x}-v_{1} \phi_{x}+u_{1} u_{1 x}+u_{2} u_{0 x}-u_{2} u_{1} \phi_{x}+12 \varepsilon^{2} \phi_{x} \phi_{t} u_{2 x}\right),
\end{aligned}
$$$$
v_{3}=\frac{1}{12 \varepsilon^{2} \phi_{x}^{2} \phi_{t}}\left(-u_{1} \phi_{x}-3 \delta^{2} u_{1 x} \phi_{x x}+\delta^{2} u_{0 x x x}-3 \delta^{2} u_{1 x x} \phi_{x}-\delta^{2} u_{1} \phi_{x x x}+v_{0 t}-v_{1} \phi_{t}\right.
$$$$
+v_{0 x} u_{2}-6 \delta^{2} \phi_{x}^{2} u_{2 x}+v_{1 x} u_{1}+v_{1} u_{1 x}+12 \varepsilon^{2} \phi_{x} \phi_{t} v_{2 x}+v_{2} u_{0 x}-\phi_{x} v_{1} u_{2}+6 \delta^{2} \phi_{x}^{3} u_{3}
$$

$\left.-\phi_{x} v_{2} u_{1}+u_{0 x}\right)$

hence Eq. (5.11) is a Bäcklund transformation too.

\section{Conclusion}

In this paper, the Painlevè test has been successfully applied to obtain new Bäcklund transformation of some of the most important nonlinear partial differential equations that have many applications in different fields. Thus the Painlevè test can be applied to different nonlinear partial differential equations to obtain new Bäcklund transformation of these partial differential equations.

\section{References}

[1]. V. O. Vakhnenko, E. J. Parkes and A. J. Morrison, A Bäcklund transformation and the inverse scattering transform method for the generalised Vakhnenko equation, Chaos, Solitons\& Fractals, 17(2003) 683-92.

[2]. M. J. Ablowitz and P. A. Clarkson, Soliton, nonlinear evolution equations and inverse scattering.New York: Cambridge University (1991).

[3]. C. S. Gardner, J. M. Green, M. D. Kruskal and R. M. Miura, Method for solving the Korteweg- de Vries equations, Phys. Rev. Lett. 19 (1967)1095-1102.

[4]. H Eichhorn, Application of the inverse scattering method to the generalized non- linear Schrodinger equation, Inverse Problems I (1985) 193-198.

[5]. C. Yong, L. Biao, and Z. Hong-Qing, B“acklund Transformation and Exact Solutions for a New Generalized Zakharov Kuzentsov Equation with Nonlinear Terms of Any Order, Commun.Theor. Phys. 39 (2003)135-140.

[6]. A. H. Khater, R. S. Ibrahim, O. H. El-Kalaawy and D. K. Callebaut, Bäcklund transformations and exact

[7]. soliton solutions for some nonlinear evolution equations of the ZS/AKNS system, Chaos, Solitons\&Fractals, 9 (1998) 18471855 .

[8]. M. F. El-Sabbagh, M. M. Hassan, E. Hamed, Bäcklund transformations for some non-linear evolution equations using Painlevè analysis, International Conference on Mathematics, The Egyptian Mathematical Society, Cairo, Egypt, 2012. R. Conte, Invariant Painleve analysis of partial differential equations, Phys. Lett.140 (1989)383- 390.

[9]. J. Weiss, M Tabor and G Carnevale, The Painleve property for partial differential equations, J. Math. Phys. 24 (1983) $522-526$.

[10]. A. Ramani, B. Grammaticos and T Bountis, The Painleve property and singularity analysis of integrable and non-integrable systems, Phys. Rep. 180 (1989) 159-245. 
[11]. M P JOY, Painleve analysis and exact solutions of two dimensional Korteweg-de Vries-Burgers equation, PRAMANAjournal of physics, 46 (1996) 1-8.

[12]. M. Y. Adamu, E. Suleiman and A. G. Madaki, Solving Nonlinear Partial Differential Equation Using Painleve Analysis, The International Journal of Engineering And Science (IJES),2(2013) 243-245.

[13]. M. A. Akbar, N. Hj. M. Ali, The modified alternative (GVG)-expansion method for finding the exact solutions of nonlinear PDEs in mathematical physics, International Journal of the Physical Sciences,6(2011) $7910-7920$.

[14]. Y. Li, D. Li, New Exact Solutions for the (2+1)-dimensional Boiti-Leon-Manna- Pempinelli Equation, Applied Mathematical Sciences, 6 (2012) $579-587$.

[15]. M. Najafi, S. Arbabi, M. Najafi, New Soliton Solutions of (2+1)-Dimensional Boiti-Leon-Manna- Pempinelli Equation, International Journal of Modern Mathematical Sciences, 6 (2013) 84-91.

[16]. A. Zamiri, The (G/G')-Expansion Method for the (2+1)-Dimensional Boiti-Leon-Manna-Pempinelli Equation, J. Basic. Appl. Sci. Res., 3(2013) 522-527.

[17]. M. Song-Hua and F. Jian-Ping, Multi Dromion-Solitoff and Fractal Excitations for (2+1)-Dimensional Boiti Leon Manna Pempinelli System, Commun. Theor. Phys., 52 (2009) 641-645.

[18]. N. Taghizadeh and S.R. Moosavi Noori, Two Reliable Methods for Solving the Modified Improved Kadomtsev-Petviashvili Equation, Appl. Appl. Math., 7(2012) $658-671$.

[19]. M. J. A. Abu Al-Shaeer, Solutions for Nonlinear Partial Differential Equations By Tan-Cot Method, IOSR Journal of Mathematics, 5 (2013) 6-11.

[20]. FU-DING XIE, Exact Solutions of Some Systems of Nonlinear Partial Differential Equations Using Symbolic Computation, Computers and Mathematics with applications, 44 (2002) 711-716. 